Fetal Diagnosis and Therapy

\title{
Impact of Quality of Nuchal Translucency Measurements on Detection Rates of Trisomies 13 and 18
}

\author{
Mark I. Evans ${ }^{a}$ Eugene Pergament ${ }^{b}$ \\ ${ }^{a}$ Comprehensive Genetics, Fetal Medicine Foundation of America, and Department of Obstetrics and Gynecology

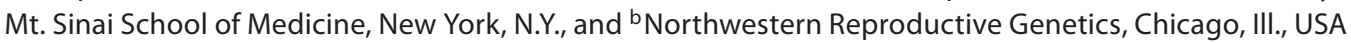

\section{Key Words}

Prenatal screening $\cdot$ Nuchal translucency $\cdot$ Trisomy $13 \cdot$

Trisomy $18 \cdot$ Biochemical screening

\begin{abstract}
Objective: To determine the relative contribution of nuchal translucency (NT) to the biochemical detection rates of combined screening for trisomies 13 and 18 in two healthcare systems practicing different quality-control systems of nuchal measurement. Methods: De-identified data collected from the Fetal Medicine Foundation in the United Kingdom and laboratory data from NTD laboratories in the United States were compared for detection rate and false-positive rate (FPR) for combined trisomies 13 and 18 screening and were subcategorized by biochemical only and biochemistry combined with NT measurement. Results: US and UK biochemical detection rates were virtually identical: $83 \%$ for a FPR of $1.8 \%$. When NT measurement was added, the US rate increased to $88 \%$ for a $0.7 \mathrm{FPR}$, but in the UK the rate increased to $94 \%$ for a FPR of $0.3 \%$. The mean NT was $1.69 \mathrm{~mm}$ in the US and $2.82 \mathrm{~mm}$ in the UK. Conclusions: NT measurement as practiced in the UK system with tight quality control significantly increased the detection rate and significantly reduced the FPR of combined screening for trisomies 13 and 18 , when compared to the US with apparently less rigid quality control.

Copyright $\odot 2010$ S. Karger AG, Basel
\end{abstract}

\section{Introduction}

Over the past 40 years, prenatal screening for aneuploidy has evolved from using maternal age alone to mid-trimester single and then multiple maternal serum markers to first-trimester biochemical markers and ultrasound measurements [1]. Each step has increased the sensitivity and specificity of aneuploidy screening. As a direct consequence, detection of aneuploid pregnancies markedly increased, while at the same time the number of pregnancies exposed to the obstetrical risk of expensive and potentially complicated procedures has been reduced [2].

In the laboratory all tests are regularly subjected to quality control to determine their reliability. It has been well established that standardization has led to markedly improved test performance [3]. For example, during the 1970 s and 1980 s, it was widely held that variability in maternal serum $\alpha$-fetoprotein (AFP) in different patient populations required that each laboratory establish their own medians and distributions. In fact, it was the considerable differences in analytical methods, rather than patient variability, that accounted for the disparity in results among laboratories $[1,2]$. Once standardized, intra-laboratory variation in the case of maternal serum AFP was actually greater than the variation between patient populations [4]. At the same time, adjustments for certain in-

\section{KARGER \\ Fax +4161306 1234 \\ E-Mail karger@karger.ch}

www.karger.com (c) 2010 S. Karger AG, Basel

$1015-3837 / 10 / 0272-0068 \$ 26.00 / 0$

Accessible online at:

www.karger.com/fdt
Mark I. Evans, MD

Comprehensive Genetics

131 E. 65 th Street

New York, NY 10065 (USA)

Tel. +1 212288 1422, Fax +1 212879 2606, E-Mail evans@ compregen.com 
dependent variables were also required in screening for aneuploidy using AFP and subsequently multiple maternal serum markers. These included gestational age, maternal weight, race, ethnic group, twins, and diabetes [5]. This process was essentially replicated when first-trimester serum markers were introduced into clinical practice.

The development of ultrasound markers identifying pregnancies at risk of aneuploidy presented a different problem. From a historical perspective, ultrasound evaluations initially were based on individual experiences, and many ultrasonographers, accomplished and otherwise, were resistant to the concept that they had to be subjected to objective external evaluation of their ultrasound measurements for purposes of quality assurance [6]. However, repeated experience has shown that if ultrasound measurements were to be used in a laboratory algorithm, quality control was essential. The net effect of failing to obtain standardization in ultrasound measurements was to lower the detection rate or unnecessarily alarm prospective parents by assessing their pregnancy as 'at increased risk' for aneuploidy [1]. Thus, the accreditation process of the Fetal Medicine Foundation (FMF) in London was created a decade ago. In one United States program that refused to participate in the accreditation process, $82 \%$ of cases were below the national median for nuchal translucency (NT) measurements, and $40 \%$ were below the 5 th percentile. In response, the skewed distribution was interpreted as reflecting 'patient population' differences rather than acknowledging that technical competence was at issue (unpublished).

More than 50,000 physicians and sonographers worldwide have now been trained by the FMF [7]. The process includes didactics, examinations, submission of films, certification, and periodic recertification $[6,7]$. In the United States, the Society for Maternal Fetal Medicine created the Nuchal Translucency Quality Review (NTQR) Program to provide education and quality review. These two programs differ in their approach to quality control, although both are based upon underlying didactic training and documentation of proper techniques and reliability of measurements. The FMF, in part by virtue of a longer history, has had a continuing reassessment and recertification process in place for nearly a decade. The NTQR is still developing its program for continual monitoring and assessment. While most of the focus of first-trimester screening has been targeted at trisomy 21 , it has long been recognized that trisomies 13 and 18 can be detected by measurement of NT and their pattern of markedly lower free $\beta$-human chorionic gonadotropin (free $\beta$-hCG) and pregnancy-associated plasma protein (PAPP-A) $[1,2,6$,
Table 1. Comparison of detection and false-positive rates in screening for trisomies 13 and 18 in the USA and UK

\begin{tabular}{llllll}
\hline & Free $\beta$-hCG and PAPP-A & & \multicolumn{2}{l}{$\begin{array}{l}\text { Free } \beta \text {-hCG and PAPP-A } \\
\text { plus nuchal translucency }\end{array}$} \\
\cline { 2 - 3 } $\begin{array}{lllll}\text { detection } \\
\text { rate, \% }\end{array}$ & $\begin{array}{l}\text { false-positive } \\
\text { rate, } \%\end{array}$ & & $\begin{array}{l}\text { detection } \\
\text { rate, } \%\end{array}$ & $\begin{array}{l}\text { false-positive } \\
\text { rate, \% }\end{array}$ \\
\hline USA & 82.3 & 1.7 & 88.4 & 0.7 \\
UK & 84.5 & 1.9 & 93.9 & 0.3 \\
\hline
\end{tabular}

7]. Here, we seek to assess any epidemiologic differences in outcomes of biochemical and NT performance between the United Kingdom and United States for the detection of trisomies 13 and 18.

\section{Methods}

De-identified data from the FMF in the United Kingdom and from the NTD (New York) laboratory databases in the United States were assessed for the performance of NT and biochemical screening in cases known to have resulted in either trisomies 13 or 18. The study periods for both groups began in 1998 and continued to 2002 for the UK and 2007 for the US as NT screening grew much faster in the UK than the US. UK data have been published, and US data were provided to the investigators by the NTD laboratories [8]. The United Kingdom data included 124 cases and the United States 66 biochemical cases, of which 42 also had NT measurements [8]. Performance of the UK and US screening programs was then compared by laboratory analysis of free $\beta$-hCG and PAPP-A with and without NT measurements. While all the UK data are from FMF accredited providers, we do not have the break down of US providers who were FMF America certified or NTQR. Data were analyzed by $\chi^{2}$ analysis and t tests as appropriate with calculation of detection rates and false-positive rates (FPRs).

\section{Results}

For both the United Kingdom and United States data, biochemical detection of trisomies 13 and 18 was virtually identical (table 1). In the United States, the sensitivity was $82.3 \%$ for a $1.7 \%$ FPR, and in the United Kingdom, it was $84.5 \%$ for a $1.9 \%$ FPR [8]. With the addition of NT, the detection rate in the United Kingdom increased to $94 \%$ with a $0.3 \%$ FPR but only to 88 for $0.7 \%$ FPR in the United States $\left(\chi^{2}=4.4, p<0.05\right)$. Multiples of median (MoM) for free $\beta$-hCG (US 0.22 vs. $0.38 \mathrm{MoM} \mathrm{UK}$ ) and PAPP-A (US 0.32 vs. $0.20 \mathrm{MoM}$ UK) were comparable between groups, but median NT measurements were sig- 
Table 2. Multiples of the median (MoM) by gestational age (GA) in the United States

\begin{tabular}{lrl}
\hline GA, weeks & $\mathrm{n}$ & MoM \\
\hline 11 & 26 & 2.14 \\
12 & 14 & 1.34 \\
13 & 2 & 1.10 \\
\hline
\end{tabular}

nificantly lower in the US data set $(1.69 \mathrm{~mm})$ compared to the UK $(2.82 \mathrm{~mm} ; \mathrm{t}=2.02, \mathrm{p}<0.05)$. NT MoM in the US trended to be lower with gestational age, but the numbers were too small to reach significance (table 2).

\section{Discussion}

First-trimester detection of trisomies 13 and 18 based on biochemistry alone demonstrated sensitivities of over $80 \%$ for a screen positive rate of $<2 \%$ and contributed a higher proportion than NT to the diagnoses of trisomies 13 and 18 when compared to that for trisomy 21 or Turner syndrome. By comparison, the 'biochemical only' Down syndrome detection rate is approximately $63 \%$ for a $95 \%$ specificity $[1,6]$. When NT was added and standardized with training and credentialing, as has been performed in the United Kingdom, the detection rate was increased to $94 \%$ with a FPR of $0.3 \%$,whereas in the United States, with the addition of NT measurements, the detection rate was lower, $88.4 \%$, with a higher FPR of $0.7 \%$.

These data are consistent with the well-established laboratory algorithm model in which rigid standardization of measurements produces the best possible performance as well as low coefficients of variation. The training model of the FMF has been published many times [6]. The applicability in the US over the last decade has been variable, and the NTQR has gone through evolutions of data collection and feedback to providers as it has become established in the US. For example, both programs required a didactic session and exam. The FMF required in addition the submission of 50 pictures of scans to be graded, and the NTQR has required 10. It has not been possible to assess the impact of differences in the number of scans submitted, or whether there are any differences in the rigor of the grading of the scans. This is a limitation of our study.

These results speak to the general proposition that the quality of a 'laboratory measurement per se' is likely to have higher performance than that of a clinically deter- mined measurement such as ultrasound [9]. We have made a similar observation in the increased birth rate of Down syndrome infants in Colorado over the last 20 years as amniocentesis and chorionic villus sampling procedures have decreased, and use of the 'genetic ultrasound' has increased [10]. The variability of the performance of ultrasound measurements parallels the experience with second-trimester estriol screening in the 1990s. Whereas the coefficients of variation (CV) for both AFP and hCG were in the range of about 7\%, many estriol assays had CVs of 15-20\%. Not surprisingly, in retrospect, those programs with tight estriol CVs showed increased performance in triple versus double screening, and those programs with poor estriol assays showed diminished performance [1]. The same principle applies to NT measurements in that tight distributions of normal data increase the likelihood that abnormal cases will be identified as such as opposed to being considered within the normal range.

The data presented here indicated better overall performance of NT measurements in the United Kingdom than in the United States. Two recent studies, one from London and another from Denmark, have corroborated a greater than $90 \%$ detection of trisomy 18 for FPRs of about $0.3 \%$ on top of the trisomy 21 FPR $[11,12]$. While is it possible to deliberate about the specific causes of the difference, the unmistakable conclusion is that better quality control appears to be in place in the United Kingdom and Denmark than in the United States. Therefore, ultrasound performance in the United States will likely continue to lag behind the United Kingdom. These data suggest that as components of first-trimester screening programs for detection of trisomies 13 and 18 are evaluated: (1) analyses of maternal serum analytes have stricter controls than ultrasound measurements and therefore more accurate assessments as to their sensitivity and specificity, and (2) programmatic differences in falsepositive and false-negative rates are likely to be a reflection of the skill and training of those performing measurements of NT. Given the much larger variability in the quality of ultrasound measurement among centers in the United States, mass screening strategies should focus on optimizing input of the highest quality variables. We have previously published that even minor underestimations of NT measurements can reduce the sensitivity of trisomy 21 detection by $18 \%$ for the same FPR [13]. Recent analysis of US NT data shows that the US distribution of measurements is still skewed to the left with considerable overrepresentation of cases $<5$ th percentile [14]. 
Currently, the more effective quality control lies in the biochemical screening parameters of free $\beta$-hCG and PAPP-A as compared to ultrasound measurement of NT. In the case of combined screening for trisomies 13 and 18 , rigid oversight of NT measurements with regular feedback, as practiced by the UK system, has demonstrat- ed an effectiveness superior to that currently in practice in the US system. The process of continual assessment with feedback, a well-established principle in business and technology assessments, has considerable applicability with regard to aneuploid screening in the first trimester [15].

\section{References}

1 Evans MI, Galen RS, Drugan A: Biochemical screening; in Evans MI, Johnson MP, Yaron Y, Drugan A (eds): Prenatal Diagnosis: Genetics, Reproductive Risks, Testing, and Management. New York, McGraw-Hill, 2006, pp 277-288.

$\checkmark 2$ Evans MI, Cuckle HS: Biochemical screening for aneuploidy. Expert Rev Obstet Gynecol 2007;2:765-773.

$>3$ O’Brien JE, Dvorin E, Drugan A, Johnson MP, Yaron Y, Evans MI: Ethnic and racial specific variation in multiple marker biochemical screening (MMBS): HCG and uE3 also require separate databases. Obstet Gynecol 1997;89:355-358.

4 Evans MI, Belsky RL, Greb AE, Dvorin E, Drugan A: Wide variation in maternal serum alpha-fetoprotein (MSAFP) reports in one metropolitan area: concerns for the quality of prenatal testing. Obstet Gynecol 1988;72:342-345.
5 Kramer RL, O’Brien JE, Critchfield G, Yaron Y, Ayoub M, Lewis PS, Johnson MP, Evans MI: The effect of adjustment of maternal serum a-fetoprotein levels in insulin-dependent diabetes mellitus Am J Med Genet 1998; 75:176-178.

6 Hyett J, Nicolaides KH: First trimester ultrasound screening with nuchal translucency; in Evans MI, Drugan A, Johnson MP, Yaron Y (eds): Prenatal Diagnosis: Genetics, Reproductive Risks, Testing and Management. New York, McGraw-Hill, 2006, pp 289-308.

7 Nicolaides KH: The 11-13+6 Weeks Scan. London, Fetal Medicine Foundation, 2004.

$>8$ Spencer K, Nicolaides KH: A first trimester trisomy 13/trisomy 18 risk algorithm combining fetal nuchal translucency thickness, maternal serum free beta-hCG and PAPP-A. Prenat Diagn 2002;22:877-879.

$\checkmark 9$ Evans MI, O’Brien JE, Dvorin E, Wapner RJ, Harrison HH: Standardization of methods reduces variability: explanation for historical discrepancies in biochemical screening. Genet Test 2003;7:81-83.

$>10$ Henry GP, Britt DW, Evans MI: Screening advances and diagnostic choice: the problem of residual risk. Fetal Diagn Ther 2008;23: 308-315.
11 Kagan KO, Wright D, Maiz N, Pandeva I, Nicolaides KH: Screening for trisomy 18 by maternal age, fetal nuchal translucency, free beta-human chorionic gonadotropin and pregnancy-associated plasma protein-A. Ultrasound Obstet Gynecol 2008;32:488-492.

12 Kirkegaard I, Petersen OB, Uldbjerg H, Tarring N: Performance of first trimester combined screening for trisomy 13 and 18 with the double test taken at a gestational age of $8+0$ to $13+6$. Prenatal Diagn 2009;29:582587.

13 Evans MI, Van Decruyes H, Nicolaides KH: Nuchal translucency (NT) measurements for first trimester screening: the 'price' of inaccuracy. Fetal Diagn Ther 2007;65:308-315.

14 Evans M, Krantz D, Hallahan T, Sherwin J: Skewed to the left: under measurement of NT's and implications for screening efficiency. Am J Obstet Gynecol 2009;201:S140.

15 Cohen AB, Hanft RS: Technology in American Health Care. Ann Arbor, University of Michigan Press, 2004. 